\title{
Obediência Infantil: Orientação Comportamental para Mães de Crianças com Dermatite Atópica
}

\author{
Jardson Fragoso Carvalho \\ Faculdade da Cidade de Salvador, Salvador, BA, Brasil \\ Departamento de Polícia Técnica da Secretaria de Segurança Pública do Estado da Bahia, \\ Salvador, BA, Brasil \\ Márcia Cristina Caserta Gon ${ }^{1}$ \\ Departamento de Psicologia Geral e Análise do Comportamento da Universidade Estadual \\ de Londrina, Londrina, PR, Brasil \\ Programa de Pós-Graduação em Análise do Comportamento da Universidade Estadual de
}

Londrina, Londrina, PR, Brasil

\section{Resumo}

Este estudo teve o objetivo de avaliar os efeitos de um programa de orientação comportamental breve para mães de crianças portadoras de dermatite atópica. Participaram quatro crianças entre 5 e 12 anos, diagnosticadas com a doença; e suas mães. As mães foram orientadas, individualmente, sobre como deveriam agir mediante comportamentos de obediência e de desobediência da criança às suas instruções para realizar o tratamento médico. A avaliação foi conduzida por meio de observação direta da interação mãe e criança, em situação de tratamento, elaborada para a finalidade da pesquisa. Os resultados mostraram que as mães seguiram as orientações dadas durante o procedimento de intervenção comportamental. Mais especificamente, o estudo mostrou que o uso de instruções diretas e de elogios descritivos, contingentes ao comportamento da criança de executar o tratamento, aumentou a frequência de obediência infantil. Estes resultados indicam que intervenções comportamentais breves podem ser eficazes na orientação do cuidador sobre como instruir crianças com dermatite atópica a realizarem tratamento médico. A instrução direta, enquanto evento antecedente, pode aumentar a probabilidade de que as crianças obedeçam seus cuidadores. Os elogios descritivos podem promover a manutenção da obediência infantil e a aprendizagem de comportamentos de cuidado com a pele.

Palavras-chave: Dermatite atópica, orientação comportamental, interação mãe-criança, obediência, infância.

\section{Child'S Compliance: Behavioral Orientation to Mothers of Children with Atopic Dermatitis}

\begin{abstract}
This study aimed to evaluate the effects of a brief behavioral orientation program for mothers of children with atopic dermatitis. The participants were four children between 5 and 12 years, diagnosed with the disease and their mothers. Mothers were instructed individually on how they should act upon behaviors

Endereços para correspondência: Universidade Estadual de Londrina, Centro de Ciências Biológicas, Departamento de Psicologia Geral e Análise do Comportamento, Campus Universitário, Rodovia Celso Garcia Cid (PR 445) km380, Gleba Palhano, Caixa Postal: 86051-980. Londrina, PR, Brasil 86051-990. E-mail: jardsonwtj@gmail.com e marciagon@sercomtel.com.br Agência de financiamento: Coordenação de Aperfeiçoamento de Pessoal de Nível Superior (Capes).
\end{abstract}


of compliance and noncompliance of children to their instructions to provide medical treatment. The evaluation was conducted through direct observation of mother and child, during treatment, prepared for the purpose of research. The results showed that mothers followed the guidance given during the procedure of behavioral intervention. More specifically, the study showed that the use of direct instruction and descriptive praise, contingent on the child's behavior to perform the treatment, increased the frequency of childlike obedience. These results indicate that brief behavioral interventions can be effective to guide caregivers how to instruct children with atopic dermatitis to undertake medical treatment. The direct instruction, while antecedent event, may increase the likelihood that children obey their caregivers. Descriptive praise may promote the maintenance of childlike obedience and learning behaviors of skin care.

Keywords: Atopic dermatitis, behavioral orientation, mother-child interaction, compliance, childhood.

\section{Obediencia Infantil: Guía de Conduta para las Madres de los Niños con Dermatitis Atópica}

\section{Resumen}

Este estudio tuvo como objetivo evaluar los efectos de un breve programa de orientación conductual para las madres de los niños con dermatitis atópica. Los participantes fueron cuatro niños entre 5 y 12 años, con diagnóstico de la enfermedad, y sus madres. Las madres fueron instruidos individualmente sobre cómo deben actuar sobre los comportamientos de la obediencia y la desobediencia de los hijos a sus instrucciones de proporcionar tratamiento médico. La evaluación se realizó a través de la observación directa de la madre y el niño, en tratamiento, preparado para el propósito de la investigación. Los resultados mostraron que las madres siguieron la orientación dada durante el procedimiento de intervención en el comportamiento. Más específicamente, el estudio mostró que el uso de la instrucción directa y la alabanza descriptiva, depende de la conducta del niño para realizar el tratamiento, el aumento de la frecuencia de la obediencia infantil. Estos resultados indican que las intervenciones conductuales breves pueden ser eficaces para guiar a los médicos cómo instruir a los niños con dermatitis atópica a realizar un tratamiento médico. La instrucción directa, en tanto que acontecimiento antecedente, puede aumentar la probabilidad de que los niños obedezcan a sus cuidadores. Elogio descriptivo puede favorecer el mantenimiento de la obediencia infantil, y el aprendizaje de conductas de cuidado de la piel.

Palabras clave: Dermatitis atópica, orientación conductual, interacción madre-hijo, obediencia, infancia.

A dermatite atópica (DA) é uma doença inflamatória da pele, de curso crônico, que tem como sintomas principais pele seca, prurido e coceira. É uma das doenças crônicas de pele mais comuns na população mundial e este número tem aumentado gradualmente, sobretudo nos países industrializados (Carroll, Balkrishnan, Feldman, Fleischer, \& Manuel, 2005; Kondo-Endo et al., 2009; Weber et al., 2008; Willians, 2005). No Brasil, a incidência de DA é de $13 \%$ entre crianças e adolescentes e, em 90\% dos casos, o início das lesões acontece durante os cinco primeiros anos de vida, existindo possibilidade de persistência na vida adulta entre 40 a $60 \%$ dos pacientes (Pires \& Cestari, 2005). O trata- mento principal para controle dos sintomas da DA, e mais comum, é o uso de corticoides para diminuir a intensidade do prurido e de emolientes e hidratantes para manter a pele sempre bem hidratada. Para alguns pacientes são indicados anti-histamínicos para conter o prurido intenso. Evitar fatores alergênicos é também fundamental (Carroll et al., 2005).

Quando se tratam de crianças com uma doença crônica, o aprendizado de comportamentos de autocuidado com a saúde depende, em especial, diretamente do seguimento das instruções de seus cuidadores bem como de seu repertório de seguir regras (Bagner, Fernandez, \& Eyberg, 2004). O não seguimento (ou desobediência) 
destas instruções pela criança levará a um aumento da probabilidade de piora dos sintomas e de recidivas (Menezes, Gon, \& Zazula, 2013).

Portanto, os cuidadores da criança que apresenta uma doença crônica são importantes agentes mediadores da aquisição dos comportamentos de autocuidado com a saúde e que deverão ser mantidos ao longo da vida de muitas delas (Amaral \& Albuquerque, 2000), como é o caso de pacientes com DA. Para eles, além da exposição constante a situações de preconceito devido à condição inestética da pele em diversos contextos sociais, os sintomas da doença provocam desconforto físico e há a necessidade de estabelecer rotinas de tratamento que nem sempre produzem efeitos imediatos e que concorrem com outras atividades consideradas mais reforçadoras para a criança (Gon, Rocha, \& Gon, 2005). Consequentemente, os cuidadores sofrem física e emocionalmente com esta rotina, sobretudo quando há resistência por parte da criança em fazer o tratamento de forma adequada (Staab et al., 2002).

Por estas razões, programas de orientação comportamental que visem ensinar os cuidadores como aumentar a probabilidade de emissão de comportamento da criança de obedecer às suas instruções para realizar o tratamento médico e cuidar da saúde e diminuir o de desobedecê-las são necessários. Isso porque baixa adesão ao tratamento prescrito pelo médico é o motivo principal de piora dos sintomas e de recidivas em pacientes com DA (Lapsley, 2006).

Programas de intervenção comportamental com crianças que apresentam problemas crônicos de saúde e/ou com seus cuidadores têm mostrado ser eficientes para aumentar a adesão ao tratamento médico e promover a melhora da qualidade de vida destas famílias (e.g., Epstein, Valoski, Wing, \& McCurley, 1990; Fornazari \& Librazi, 2009; Soares, Trassi, Pacífico, \& Inácio, 2014; Staab et al., 2002).

O objetivo do presente estudo foi o de avaliar os efeitos de um programa de orientação comportamental breve para mães sobre os comportamentos de obediência e desobediência de crianças com DA quanto ao uso de medicamentos tópicos.

\section{Método}

\section{Participantes}

Quatro díades formadas pela mãe e sua criança. As crianças tinham 5 anos (D1), 7 anos (D3 e D4) e 12 anos (D2), sendo três do gênero masculino (D1, D2 e D4) e uma do gênero feminino (D3). Os critérios para inclusão das crianças foram: (a) ter idade entre cinco e 12 anos; (b) possuir diagnóstico de DA; (c) estar em tratamento médico durante o período de coleta de dados; (d) não estar em atendimento psicológico ou psicoeducacional; (e) o cuidador relatar desobediência infantil. Para os cuidadores, o critério de inclusão foi o de apresentar repertório de leitura (alfabetização).

Projeto aprovado pelo Comitê de Ética em Pesquisa Envolvendo Seres Humanos da Universidade Estadual de Londrina (processo 12145/2011 e parecer 117/2011).

\section{Procedimento}

As mães foram entrevistadas individualmente para levantamento de informações sobre seu comportamento e da criança em relação à doença e ao tratamento médico. Na condição de avaliação pré-intervenção (Linha de Base, LB), após um período de 10min de adaptação da díade à sala e à filmadora, a mãe, que havia sido previamente orientada pelo pesquisador, deveria realizar o tratamento médico juntamente com a criança da forma como o fazia em casa. O medicamento tópico (pomada ou creme) deveria ser passado em, pelo menos, três partes do corpo (e.g., braço, pescoço e mão).

A etapa de intervenção foi composta de quatro encontros individuais de aproximadamente $120 \mathrm{~min}$, realizados semanalmente e nos quais foram apresentados e discutidos os princípios da Análise do Comportamento e aqueles propostos na Interação Direcionada aos Pais (PDI) de Eyberg (1988) que são a manipulação direta de eventos antecedentes (tipo de instruções) e de consequentes (Elogios e time-out). No presente estudo, a situação de brincadeira usada no PDI foi substituída pela de tratamento tópico para DA. Foram realizadas duas sessões 
de avaliação (A1 e A2) sobre manipulação de eventos antecedentes e consequentes, ao término das segunda e terceira sessões de intervenção, respectivamente. Uma sessão de avaliação pós-intervenção (Avaliação Final, AVF) foi conduzida seguindo-se o mesmo procedimento da etapa de avaliação pré-intervenção. Os objetivos de cada uma das sessões de intervenção estão descritos na Tabela 1.

\section{Tabela 1}

\section{Objetivos das Sessões de Intervenção}

Sessões Objetivos

1. Apresentar a proposta do estudo.

2. Explicar o que caracteriza uma doença como sendo crônica e porque é importante aderir ao tratamento médico prescrito.

3. Explicar porque é importante que a criança apresente comportamentos de autocuidado, em especial com a sua pele.

4. Apresentar o conceito de comportamento segundo a Análise do Comportamento e as funções do sistema Antecedent-Behavior-Consequence (ABC).

1. Estimar a importância do contexto na emissão de respostas de obediência das crianças.

2. Demonstrar a importância dos eventos antecedentes na emissão do comportamento.

3. Explicar o estado motivacional dos pais como uma variável que faz parte da interação e como pode se relacionar à probabilidade de emissão de comportamentos de obediência e de desobediência infantil no momento do tratamento.

4. Explicar o que é uma instrução direta e uma instrução indireta, dar exemplos, e examinar como elas podem promover a aprendizagem do comportamento de obediência infantil.

5. Demonstrar como deve ser apresentada uma instrução direta por meio das três regras de Eyberg (1988).

1. Demonstrar como as consequências quando contingentes ao comportamento de obediência e de desobediência podem alterar sua probabilidade de emissão futura.

2. Explicar o que é reforçamento positivo e negativo.

3. Explicar a importância da consequenciação positiva contingente ao comportamento de obedecer infantil e demonstrar como o elogio descritivo aumenta a probabilidade de sua emissão em situações semelhantes.

4. Explicar a importância de ignorar os comportamentos inadequados, desde que não apresentem riscos à integridade física ou moral da criança.

5. Explicar o que é o time-out e como utilizá-lo como procedimento de controle do comportamento de desobediência (estabelecimento de limites) como alternativa para a punição física ou verbal.

1. Integrar os conteúdos apresentados e discutidos durante as sessões anteriores e possibilitar uma revisão por meio de exemplos de aplicações práticas.

\section{Categorização dos Comportamentos da Díade Mãe-Criança, Registro e Análise dos Dados}

Os comportamentos infantis registrados foram: obediência (iniciar a realização de uma tarefa, a partir da instrução da mãe, em até $6 \mathrm{~s}$ e concluí-la); desobediência (não iniciar a tarefa em até $6 \mathrm{~s}$, a partir da instrução da mãe ou não concluí-la) e inadequados (emissão de respostas verbais ou não verbais que pudessem ter função de fuga ou esquiva da tarefa, e.g., gritar, chorar, suspirar, resmungar ou tentar sair da sala). Os comportamentos maternos registrados foram: instrução direta (verbalização de uma ordem, pedido ou exigência de modo claro, simples e direto visando indicar o comportamento esperado da criança, e.g., "Passe o creme no seu braço", "Passe óleo no seu cotovelo"); instrução indireta (verbalização de uma ordem, exigência ou direcionamento de modo não específico, ambíguo ou hesitante para a emissão de uma resposta comportamental, e.g,"Precisa passar 
óleo?”, “A pomada não chegou sozinha aqui!", "Quem vai buscar a pomada?"); elogios descritivos (elogiar, descrevendo o comportamento da criança, e.g., "Que bom que você está passando a pomada no rosto!", "Como você passou bem o creme no joelho!"); repreensão verbal (repreensão em relação ao comportamento de desobedecer e/ou inadequado da criança, de modo verbal,e.g., "Não pode xingar a mãe!", “Assim você vai se machucar e vai sair sangue da sua perna") e orientação física (dirigir a criança fisicamente para a emissão de comportamentos de obediência em relação à tarefa, e.g., puxá-la pela mão, buscá-la em um canto da sala, colocar o tubo de pomadas em sua mão e abri-lo juntamente com a criança).

As frequências dos comportamentos da mãe e da criança foram comparadas nas quatro etapas. Dois juízes, previamente treinados, assistiram, de forma independente, às filmagens e registraram os comportamentos emitidos pela díade, de acordo com categorias previamente de- finidas, em intervalos parciais de $6 \mathrm{~s}$. O critério de concordância adotado entre os observadores foi o de $85 \%$.

\section{Resultados}

$\mathrm{Na}$ LB, a criança D1 apresentou $100 \%$ de desobediência e $50 \%$ de inadequados e a mãe apresentou $100 \%$ de instrução indireta. Na condição A1, a criança emitiu 75\% de obediência e $43 \%$ de inadequados e a mãe apresentou $80 \%$ de instruções diretas, $20 \%$ de instruções indiretas e $60 \%$ de orientação física. Na condição $\mathrm{A} 2$, a criança apresentou $75 \%$ de obediência e $20 \%$ de inadequados. A mãe apresentou $75 \%$ de instruções diretas e de orientação física. A obediência infantil foi consequenciada com elogio pela mãe em 25\% das vezes. Durante AVF, a criança obedeceu $100 \%$ mas apresentou $25 \%$ de inadequados enquanto comportava-se deste modo. A mãe apresentou $83 \%$ de instruções diretas, 33\% de orientação física e $67 \%$ de elogios.
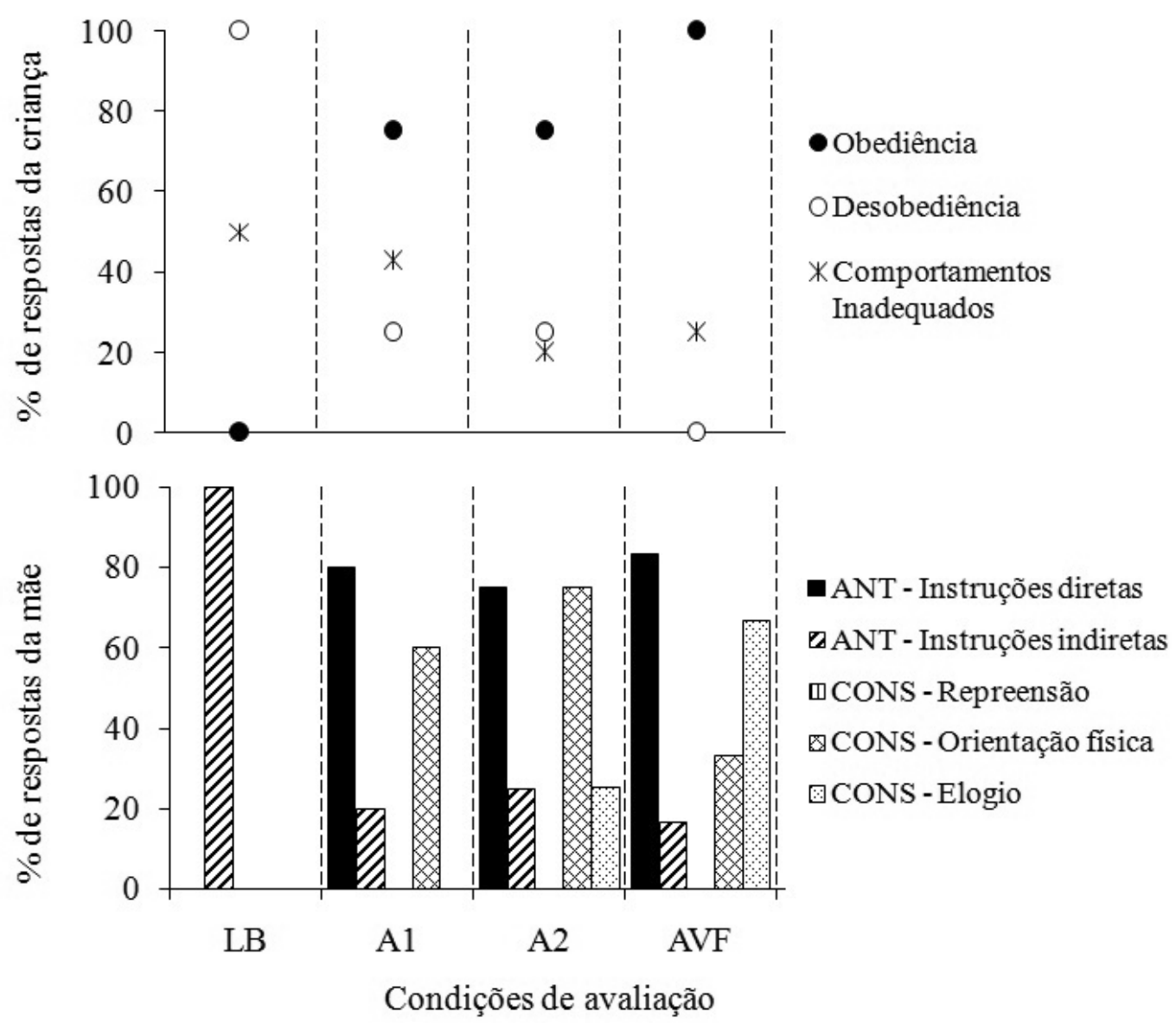

Figura 1. Porcentagens de respostas avaliadas na interação da díade mãe-criança D1. 
A criança D2 apresentou na LB 75\% de obediência e $50 \%$ de inadequados e a mãe $75 \%$ de instruções indiretas, $25 \%$ de instruções diretas, $25 \%$ de repreensão verbal e $75 \%$ de orientação física. Na condição A1 esta criança apresentou $100 \%$ de obediência e $20 \%$ de inadequados e a mãe a instruiu diretamente em $100 \%$ das vezes. Durante a condição A2, a criança apresentou
$85 \%$ de obediência e $12,5 \%$ de inadequados e a mãe, $100 \%$ de instruções diretas, $49 \%$ de repreensões verbais, $15 \%$ de orientação física e $42,8 \%$ de elogios. Durante a AVF, a criança apresentou $100 \%$ de obediência e não emitiu inadequados. Esta mãe utilizou 83,3 \% de instruções diretas, $16,7 \%$ de instruções indiretas, $16,7 \%$ de repreensões verbais e $33,3 \%$ de elogios.
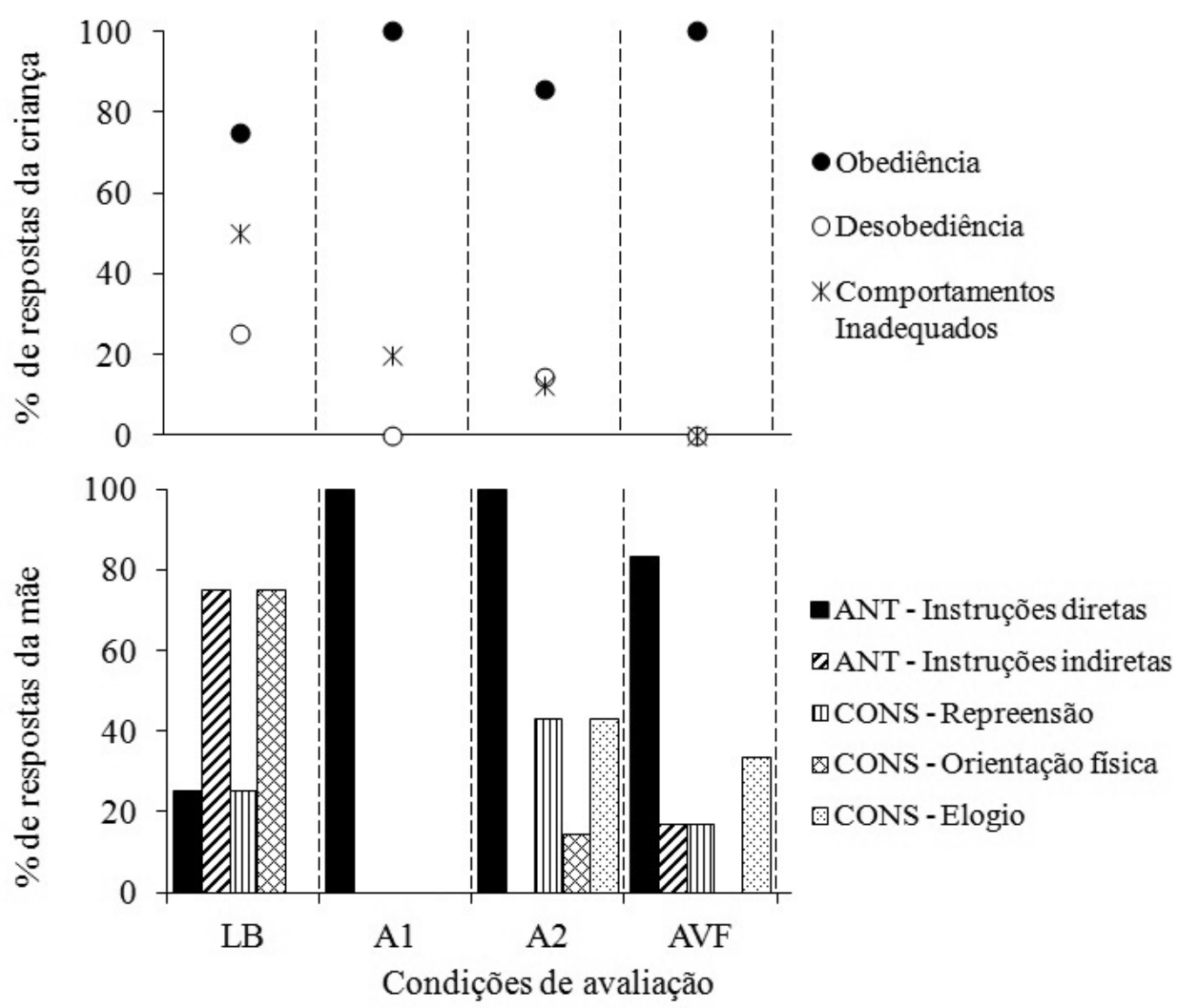

Figura 2. Porcentagens de respostas avaliadas na interação da díade mãe-criança D2.

A criança D3, na condição de LB, apresentou $66,7 \%$ de obediência e $33,3 \%$ de inadequados e a mãe, $83,3 \%$ de instruções diretas e $66,7 \%$ de orientação física. Durante a condição A1, a criança obedeceu $100 \%$ das solicitações maternas e exibiu $25 \%$ de inadequados e a mãe utilizou-se de instrução direta em $100 \%$ das vezes. Na condição $\mathrm{A} 2$, a criança obedeceu $100 \%$ das instruções da mãe e não apresentou comportamento inadequado. A mãe, por sua vez, apresentou $100 \%$ de instruções diretas e consequenciou o comportamento de obedecer da criança com elogio em $50 \%$ das vezes. $\mathrm{Na}$ etapa AVF, a criança manteve os $100 \%$ de apresentação de comportamento de obedecer como observado na LB, não desobedecendo, portanto, às instruções dadas pela mãe. Esta instruiu diretamente o comportamento da criança de realizar as tarefas do tratamento em $100 \%$ das vezes e consequenciou $80 \%$ dos comportamentos de obediência com elogios. 

com Dermatite Atópica.

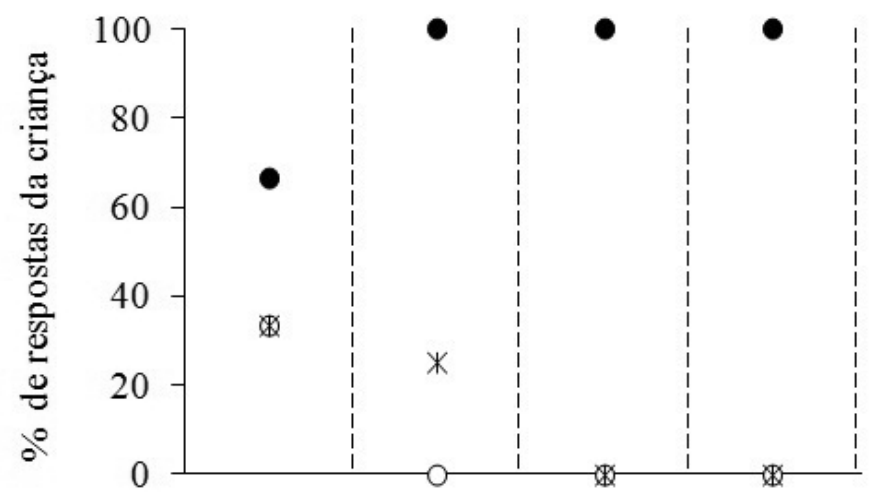

- Obediência

ODesobediência

* Comportamentos Inadequados

-ANT - Instruções diretas घANT - Instruções indiretas ๓CONS - Repreensão ⿴囗十 CONS - Orientação física 웅 CONS - Elogio

Figura 3. Porcentagens de respostas avaliadas na interação da díade mãe-criança D3.

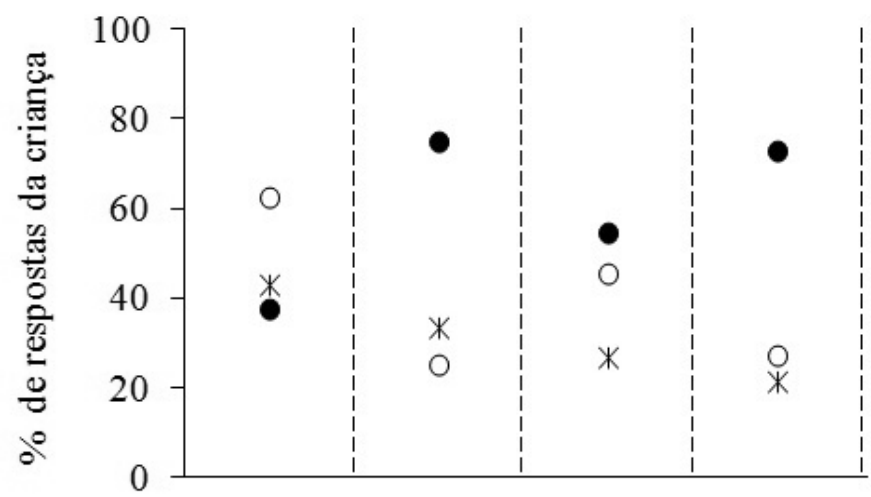

- Obediência

ODesobediência

※ Comportamentos Inadequados

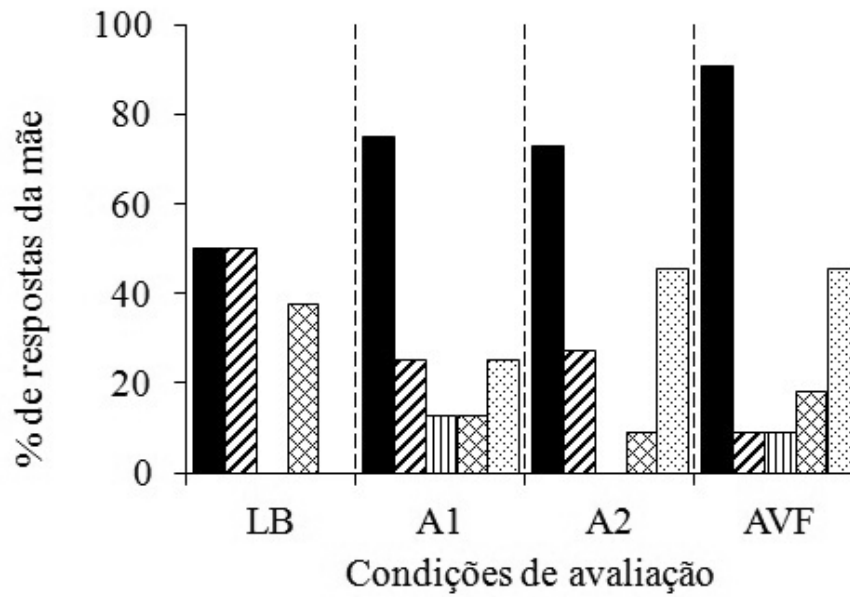

- ANT - Instruções diretas $\triangle \mathrm{ANT}$ - Instruções indiretas ๓CONS - Repreensão ⿴囗十 CONS - Orientação física 은 - Elogio

Figura 4. Porcentagens de respostas avaliadas na interação da díade mãe-criança D4. 
A criança D4, na etapa de LB, exibiu 37,5\% de obediência e $42,9 \%$ de inadequados e a mãe, $50 \%$ de instrução direta e $37,5 \%$ de orientação física. Na condição A1, a criança obedeceu $75 \%$ e exibiu $33,3 \%$ de inadequados e a mãe $75 \%$ de instrução direta, $12,5 \%$ de repreensão verbal e $25 \%$ de elogios. Durante a condição A2, a obediência infantil foi de $54,5 \%$ e inadequados de $24,7 \%$, por sua vez, a mãe apresentou $72,3 \%$ de instrução direta, $45 \%$ de elogios e $9,1 \%$ de orientação física. Na etapa AVF, esta criança obedeceu $72,7 \%$ e apresentou $21,4 \%$ de inadequados. A mãe emitiu $90 \%$ de instrução direta, $9,1 \%$ de repreensão verbal, $18,2 \%$ de orientação física e $45 \%$ de elogios.

\section{Discussão}

Os resultados encontrados para as quatro díades mostraram que o aumento da porcentagem de emissão de instruções diretas pelas mães resultou em aumento na porcentagem de respostas de obediência infantil. Este resultado corrobora aquele de Zazula (2011) que analisou a relação do tipo de instrução com o comportamento de obedecer de crianças com DA. Segundo Kalb e Loeber (2003), instruções diretas que descrevem o comportamento esperado da criança, estão mais relacionadas com o comportamento de obediência do que instruções apresentadas de maneira vaga. Stephenson e Hanley (2010) pontuam que além de dar instruções diretas, instruir a criança sobre o que ela deve fazer ao invés do que "não deve" por meio de comandos simples (um por vez), tem demonstrado ser eficaz para que a obediência infantil aconteça. Esta discussão está de acordo também com as propostas de Bell e Eyberg (2002) e Eyberg (1988) segundo as quais ensinar os pais formas diretas de instruir a criança para executar uma tarefa aumentam as chances de que ela os obedeça.

A díade que melhor representa a mudança de comportamento de obedecer da criança na medida em que a mãe passa a usar instruções diretas é a D1, de cinco anos de idade. Autores como Blum, Williams, Friman e Christophersen (1995), Bueno, Santos e Moura (2010) e Stephenson e Hanley (2010) afirmam que, quanto menor é a criança, o desenvolvimento do repertório verbal pode limitar o comportamento de obedecer às instruções dadas pela mãe. Em virtude disso é provável, portanto, que após a mãe ser orientada sobre como instruir diretamente o comportamento da criança de executar as tarefas de tratamento médico e dar um comando de cada vez, houve um aumento crescente na porcentagem de obediência infantil, atingindo $100 \%$ na etapa AVF. No contexto da saúde, observa-se que prescrições médicas podem envolver cadeias comportamentais complexas ou mesmo simples, mas a serem repetidas por um período de tempo prolongado. Por esta razão, a instrução do cuidador sendo apresentada diretamente, de modo sistemático, em partes e continuamente, pode diminuir o grau de complexidade da tarefa e com isso, aumentar a probabilidade da criança segui-la, sobretudo as mais novas (Luciano \& Herruzo, 1992). Além disso, como discutido por Sartor (2010), uma criança com cinco anos de idade não teria ainda as habilidades motoras e cognitivas suficientemente desenvolvidas de forma a entender e realizar a tarefa de conduzir o tratamento tópico sozinha (passar a pomada sozinha, manusear a medicação), necessitando da ajuda de um adulto para isso, como pode ser o caso de D1.

Apenas a mãe de D3 já apresentava repertório de instrução direta. Uma vez que este repertório já estava instalado, as orientações do pesquisador poderiam ter a função de fortalecê-lo e ao mesmo tempo diminuir a porcentagem de orientação física e de instruções indiretas que deixaram de ocorrer a partir da primeira sessão. Esta probabilidade mais elevada de a mãe responder discriminativamente às orientações do pesquisador e aos comportamentos apresentados pela criança durante as sessões de avaliação poderia explicar a porcentagem de respostas de obediência da criança que foi a mais elevada quando comparada às das demais díades.

Quanto à análise dos eventos consequentes ao comportamento de obediência (elogio descritivo) e de desobediência infantil (time-out), os resultados obtidos na LB e na A1 mostraram que três das mães não apresentaram elogios contingentes à execução das tarefas e que apenas a 
mãe D4 o fez em porcentagem baixa. Este resultado corrobora os de Zazula (2011) que mostrou ausência de elogios, pelas mães, ao comportamento de obediência das crianças. Contudo, no presente estudo, após as mães serem orientadas a consequenciar com elogios descritivos o comportamento de obediência, as crianças passaram a seguir suas instruções (condição A2 e AVF). A modelagem por meio de reforçamento positivo é amplamente relatada na literatura analítico comportamental como importante estratégia para aquisição, manutenção e fortalecimento de comportamentos específicos (Sidman, 1989/1995; Skinner, 1953/2003). No caso das crianças com DA, a aquisição de novos repertórios de autocuidado sendo consequenciada por elogio se torna importante, uma vez que a ação de cuidar da pele não será seguida de melhora imediata dos sintomas ou cura da doença. Em razão disso, Ferreira (2006) e Malerbi (2000) pontuam que o controle social, sobretudo o dos familiares, é muito importante para a adesão ao tratamento em doenças crônicas já que as respostas de autocuidado devem ser constantes a despeito dos resultados imediatos.

Ainda na discussão dos resultados da manipulação de eventos consequentes está a ausência do time-out, usado para controlar a desobediência infantil. Uma hipótese para explicar a não utilização deste procedimento pelas mães pode ser o aumento da emissão de instruções diretas (evento antecedente) e de elogios descritivos (evento consequente), levando a um aumento da probabilidade de que as crianças obedecessem às instruções. Desse modo, o uso de time-out como forma de impor limites à criança (Jacovozzi, 2009), restringindo a apresentação de comportamentos de desobediência, não foi necessário.

Um dado a ser destacado é o de emissão de comportamentos de orientação física pelas mães. Três das quatro mães (D2, D3 e D4) utilizaram-se da orientação física durante a LB como forma de ajudar na execução da tarefa de passar a pomada no corpo pela criança. De acordo com Kern, Delaney, Hilt, Bailin e Elliot (2002), a orientação física é geralmente utilizada como estratégia auxiliar para aumentar os comportamentos de obediência da criança após a instrução ser solicitada. Contudo, por estar relacionada com a impossibilidade de fuga da criança, esta estratégia pode ser reforçadora para a mãe, mas aversiva para a criança, sobretudo para as mais velhas, o que poderá aumentar a probabilidade de que elas passem a emitir comportamentos inadequados (Piazza et al., 2003). Assim, para estas três díades, uma hipótese que pode ser testada em estudo posterior é a de que a orientação física possa relacionar-se à impossibilidade da criança fugir da situação.

\section{Considerações Finais}

O presente estudo mostrou que intervenções comportamentais podem ser eficientes para aquisição de comportamentos do cuidador que aumentem a probabilidade de a criança obedecer as suas instruções para realizar o tratamento médico. Contudo, a falta de avaliação de seguimento (follow-up) não permite afirmar que esta aprendizagem foi mantida pelas mães ao longo do tempo. Fazem-se necessários estudos posteriores que apresentem em seu método este tipo de avaliação. Ainda, hipóteses que foram levantadas pelos pesquisadores poderão ser investigadas visando o aprimoramento do procedimento, em especial considerando-se maior homogeneidade para critério de inclusão dos participantes, como por exemplo, a idade da criança, localização das lesões e a severidade dos sintomas.

\section{Referências}

Amaral, V. L. R., \& Albuquerque, S. R. T. P. (2000). Crianças com problemas crônicos de saúde. In E. F. M. Silvares (Ed.), Estudos de caso em Psicologia Clínica Comportamental Infantil (pp. 219-233). Campinas, SP: Papirus.

Bagner, D. M., Fernandez, M. A., \& Eyberg, S. M. (2004). Parent-child interaction Therapy and chronic illness: A case study. Journal of Clinical Psychology in Medical Settings, 11(1), 1-6. doi:10.1023/B:JOCS.0000016264.02407.fd

Bell, S., \& Eyberg, S. M. (2002). Parent-child interaction therapy. In S. L. VandeCreek, S. Knapp, \& T. L. Jackson (Eds.), Innovations in clinical practice: A source book (Vol. 20, pp. 57-74). Sarasota, FL: Professional Resource Press. 
Blum, N. J., Williams, G. E., Friman, P. C., \& Christophersen, E. R. (1995). Disciplining children: Verbal instructions and reasoning. Pediatrics, 96(2), 336-341.

Bueno, A. C. W., Santos, B. C., \& Moura, C. B. (2010). Obediência infantil: Conceituação, medidas comportamentais e resultados de pesquisas. Psicologia: Teoria e Pesquisa, 26(2), 203216.

Carroll, C. L., Balkrishnan, R., Feldman, S. R., Fleischer, A. B., Jr., \& Manuel, J. C. (2005). The burden of atopic dermatitis: Impact on the patient, family, and society. Pediatric Dermatology, 22(3), 192-199. doi:10.1111/j.15251470.2005.22303.x

Epstein, L. H., Valoski, A., Wing, R. R., \& McCurley, J. (1990). Ten-year follow-up of behavioral, family-based treatment for obese children. Journal of the American Medical Association, 264(19), 2519-2523.

Eyberg, S. (1988). Parent-child interaction therapy: Integration of traditional and behavioral concern. Child \& Family Behavior Therapy, 10(1), 33-46. doi:10.1300/J019v10n01_04

Ferreira, E. A. P. F. (2006). Adesão ao tratamento em Psicologia Pediátrica. In M. A. Crepaldi, M. B. M. Linhares, \& G. B. Peroza (Eds.), Temas em Psicologia Pediátrica (pp. 112-144). São Paulo, SP: Casa do Psicólogo.

Fornazari, S. A., \& Librazi, L. M. (2009). Redução de comportamentos aberrantes em crianças com deficiência mental severa ou múltipla utilizando o procedimento de reforçamento diferencial de outros comportamentos (DRO). In E. J. Manzini, M. C. Marquezine, R. M. Busto, E. D. O. Tanaka, \& D. S. Fujisawa (Eds.), Procedimentos de ensino e avaliação em Educação Especial: Estudos multidisciplinares em Educação Especial (pp. 49-60). Londrina, PR: Editora da Universidade Estadual de Londrina.

Gon, M. C. C., Rocha, M. M., \& Gon, A. S. (2005). Análise do conceito de estigma em crianças com dermatoses crônicas. Revista Brasileira de Terapia Comportamental e Cognitiva, 7(1), 15-20.

Jacovozzi, F. M. (2009). Procedimentos aversivos: Divergências entre recomendações de Skinner e de Sidman e de psicoterapeutas infantis (Dissertação de mestrado não publicada, Programa de Pós-Graduação em Análise do Comportamento, Universidade Estadual de Londrina, PR, Brasil).
Kalb, L. M., \& Loeber, R. (2003). Child disobedience and noncompliance: A review. Pediatrics, 111, 641-652. doi:10.1542/peds.111.3.641

Kern, L., Delaney, B. A., Hilt, A., Bailin, D. E., \& Elliot, C. (2002). An analysis of physical guidance as reinforcement for noncompliance. Behavior Modification, 26, 516-536. doi:10.1177/0145445502026004005

Kondo-Endo, K., Ohashi, Y., Nakagawa, H., Katsunuma, T., Ohya, Y., Kamibeppu, K., \& Masuko, I. (2009). Development and validation of a questionnaire measuring quality of life in primary caregivers of children with atopic dermatitis (QPCAD). British Journal of Dermatology, 161, 617-625. doi:10.1111/j.13652133.2009.09177.x

Lapsley, P. (2006). The double benefits of educational programmers for patients with eczema. British Medical Journal, 332(936), 923-924.

Luciano, M. C., \& Herruzo, J. (1992). Some relevants components of adherence behavior. Journal of Behavior Therapy and Experimental Psychiatry, 23(2), 117-124. doi:10.1016/00057916(92)90009-8

Malerbi, F. E. (2000). Adesão ao tratamento. In R. R. Kerbauy, Sobre comportamento e cognição (pp. 148-153). Santo André, SP: Esetec.

Menezes, C. C., Gon, M. C., \& Zazula, R. (2013). Análise funcional de eventos antecedentes ao comportamento de desobediência de crianças. Psicologia: Teoria e Prática, 15(2), 19-32.

Piazza, C. C., Fisher, W. W., Brown, K. A., Shore, B. A., Patel, M. R., Katz, R. M., ...Blakely-Smith, A. (2003). Functional analysis of inappropriate mealtime behaviors. Journal of Applied Behavior Analysis, 36(2), 187-204. doi:10.1901/ jaba.2003.36-187

Pires, M. C., \& Cestari, S. C. P. (2005). Dermatite atópica. Rio de Janeiro, RJ: Diagraphic.

Sartor, M. S. (2010). Análise funcional do comportamento de desobediência ao tratamento médico de crianças com dermatite atópica (Dissertação de mestrado não publicada, Programa de Pós-Graduação em Análise do Comportamento, Universidade Estadual de Londrina, PR, Brasil).

Sidman, M. (1995). Coerção e suas implicações (R. Azzi \& M. A. Andery, Trads.). Campinas, SP: Editorial Psy. (Original publicado em 1989)

Skinner, B. F. (2003). Ciência e comportamento hu- 
mano (J. Todorov \& R. Azzi, Trads.). São Paulo, SP: Martins Fontes. (Original publicado em 1953)

Soares, M. R. Z., Trassi, A. P., Pacífico, C. F., \& Inácio, F. F. (2014). Manejo comportamental de crianças com câncer: Uma proposta de intervenção em grupo com cuidadores. In E. M. F. Seidl \& M. C. O. S. Miyazaki (Eds.), Psicologia da Saúde: Pesquisa e atuação profissional no contexto de enfermidades crônicas (pp. 225-244). Curitiba, PR: Juruá.

Staab, D., von Ruedden, U., Kehrt, R., Erhart, M., Wenninger, K., Kamtsiuris, P., \& Wahn, U. (2002). Evaluation of a parental training program for the management of childhood topic dermatitis. Pediatry Allergy Immunology, 13, 84-90.

Stephenson, K. M., \& Hanley, G. P. (2010). Preschoolers' compliance with simple instructions: A descriptive and experimental evaluation. Journal of Applied Behavior Analysis, 43, 229. 247. doi:10.1901/jaba.1975.8-332
Weber, M. B., Fontes, P. T. L., Neto, Prati, C., Soirefman, M., Mazzotti, N. G., Barzenski, B., \& Cestari, T. F. (2008). Improvement of pruritus and quality of life of children with atopic dermatitis and their families after joining support groups. Journal European Academy of Dermatology and Venereology, 22, 992-997.

Willians, H. C. (2005). Atopic dermatitis. The New England Journal of Medicine, 22(352), 23142324. doi:10.1056/NEJMcp042803

Zazula, R. (2011). Obediência de crianças com dermatite atópica às instruções do cuidador para realizar tratamento médico (Dissertação de mestrado não publicada, Programa de Pós-Graduação em Análise do Comportamento, Universidade Estadual de Londrina, PR, Brasil).

Recebido: $28 / 04 / 2014$

$1^{a}$ revisão: $21 / 08 / 2014$ Aceite final: 21/08/2014 